\title{
Aplicação de lodos de estações de tratamento de água e de tratamento de esgoto em solo degradado
}

\author{
Application of sludges from water treatment plant and from sewage \\ treatment plant in degraded soil
}

\begin{abstract}
Simone Bittencourt
Doutoranda em Engenharia de Recursos Hídricos e Ambiental pela Universidade Federal do Paraná (UFPR). Analista da Companhia de Saneamento do Paraná (SANEPAR) - Curitiba (PR), Brasil.

Beatriz Monte Serrat

Doutora em Solos e Nutrição de Plantas pela Universidade de São Paulo (USP). Professora Sênior do Programa de Pós-Graduação em Ciência do Solo da UFPR - Curitiba (PR), Brasil.

Miguel Mansur Aisse

Doutor em Engenharia Civil pela USP. Professor do Programa de Pós-Graduação em Engenharia de Recursos Hídricos e Ambiental da UFPR - Curitiba (PR), Brasil.
\end{abstract}

\author{
Lia Márcia Kugeratski de Souza Marin \\ Mestre em Geologia Ambiental pela UFPR - Curitiba (PR), Brasil. \\ Caio César Simão \\ Engenheiro Agrônomo pela UFPR - Curitiba (PR), Brasil.
}

\section{Resumo}

O objetivo deste estudo foi avaliar o efeito da aplicação de lodo de Estação de Tratamento de Água (ETA) em solo degradado, com presença e ausência de Iodo de Estação de Tratamento de Esgoto (ETE), na produtividade do milheto e nas características de fertilidade desse solo. A área experimental foi resultante de depósito de materiais oriundos da construção da ETE Padilha Sul, em Curitiba (PR). Os blocos casualizados foram arranjados em parcelas subdivididas e uma testemunha, sendo a parcela principal constituída por ausência e presença de lodo de ETE (77 Mg.ha-1 Sólidos Totais - ST) e a subparcela por doses de lodo de ETA (24, 37 e 61 Mg.ha-1 (ST)). Concluiu-se que a aplicação de lodo de ETA não teve efeito sobre a produtividade de milheto, tampouco sobre os teores dos elementos avaliados no solo. No entanto, na presença do lodo de esgoto, a sua aplicação foi favorável à dinâmica do nitrogênio do solo até à dose de $37 \mathrm{Mg}$.ha-1 e a aplicação do lodo de ETE neutralizou o alumínio trocável, elevou o pH, o cálcio, carbono, fósforo e a saturação de bases, e reduziu a acidez potencial do solo.

Palavras-chave: destinação final; área degradada; lodo de esgoto; aplicação de lodos.

\begin{abstract}
The objective of this study was to evaluate the effect of Sludge Water Treatment Plant (WTP) in degraded soil in the presence and absence of sludge Sewage Treatment Plant (STP), in the millet productivity and in the soil fertility characteristics. The experimental area was due to materials deposit from the construction of ETE Padilha Sul, in Curitiba, Paraná, Brazil. The randomized blocks were arranged in split plots and a witness, being the main plot, constituted by the absence and presence of STP sludge (77 Mg.ha-1 Total Solids - TS) and subplot by doses of WTS (24, 37 and $61 \mathrm{Mg}^{-h^{-1}} \mathrm{TS}^{-}$). It was concluded that the application of WTS had no effect on the productivity of millet, nor about the proportion of elements evaluated in the soil. However, in the presence of sewage sludge, its application was favorable to the dynamic of nitrogen until the dose $37 \mathrm{Mg}_{\text {.ha- }}{ }^{-1}$ and the application of sludge STP neutralized the exchangeable aluminum, increased the pH, calcium, carbon, phosphorus and base saturation, and reduced the potential soil acidity.
\end{abstract}

Keywords: disposal; degraded area; sewage sludge; mud application. 


\section{Introdução}

Atualmente, um dos desafios para as empresas de saneamento é a adoção de alternativas adequadas para a destinação final do lodo gerado nas Estações de Tratamento de Água (ETA) sob os aspectos: econômico, técnico e ambiental. Este resíduo sólido (ASSOCIAÇÃO BRASILEIRA DE NORMAS TÉCNICAS, 2004) é gerado no processo de transformação da água bruta, captada principalmente em rios e reservatórios, em água potável para consumo humano.

Composto por substâncias sólidas, orgânicas e inorgânicas, provenientes da água bruta e de coagulantes e floculantes utilizados no tratamento (GRANDIN et al., 1993; SILVA et al., 2000), o lodo de ETA é removido periodicamente dos decantadores e filtros para garantir a eficiência do tratamento de água, que, em geral, segue as seguintes etapas: coagulação, floculação, decantação e filtração.

Entre as alternativas de disposição final tem-se a incorporação em materiais de construção civil (SOUZA, 2010), a disposição em aterros sanitários e a aplicação controlada no solo (MOTTA et al., 2005). Esta última em expansão, visto que os compostos em maior proporção no lodo de ETA, os óxidos e hidróxidos de alumínio e ferro, argilas silicatadas e matéria orgânica (AWWA, 1990), são constituintes de solo. No entanto, para que esta prática seja considerada uma alternativa viável, faz-se necessário comprovar que não cause impactos negativos no solo receptor.

Avaliações quanto ao efeito do alumínio (Al) são necessárias, uma vez que ele pode reduzir a disponibilidade de fósforo às plantas e/ ou, em excesso, causar efeito tóxico à maioria das plantas cultivadas (MOTTA et al., 2005).

No Distrito Federal, desde março de 1997, o lodo proveniente da lavagem de filtros da ETA Rio Descoberto, que utiliza o coagulante sulfato de alumínio férrico, é centrifugado e encaminhado para recuperação de área degradada de cascalheira desativada (BARBOSA, 1997). Em estudo nesta área degradada, Moreira et al. (2009) verificaram que a aplicação do lodo promoveu a imobilização do $\mathrm{Al}$ trocável e do chumbo $(\mathrm{Pb})$ anteriormente disponíveis no solo, bem como promoveu a transferência de nutrientes para os horizontes mais profundos do solo, permitindo a fixação da vegetação. Segundo Moreira et al. (2011), o lodo de ETA pode ser considerado um resíduo não inerte e compatível com o uso em recuperação de áreas degradadas em regiões com características geológicas e hidroquímicas similares às da área de cascalheira desativada, onde o estudo foi realizado.

A utilização do lodo de ETA na recuperação de áreas degradadas pode ser otimizada pela aplicação conjunta de um resíduo orgânico (TEIXEIRA et al., 2005), como o lodo gerado em Estações de Tratamento de Esgoto (ETE), material de elevado potencial agronômico, rico em matéria orgânica e nutrientes, como nitrogênio e fósforo (PEDROZA et al., 2006; TAMANINI et al., 2008). O lodo de esgoto, quando higienizado por processo alcalino, apresenta também potencial corretivo da acidez de solo (SERRAT et al., 2011).
O objetivo do presente estudo foi avaliar o efeito da aplicação de lodo de ETA em solo degradado, na presença e ausência de lodo de ETE, na produtividade do milheto e nas características de fertilidade desse solo.

\section{Metodologia}

A pesquisa foi desenvolvida em 3 mil $\mathrm{m}^{2}$ de uma área externa da ETE Padilha Sul, da Companhia de Saneamento do Paraná (SANEPAR), no município de Curitiba (PR). A degradação do local foi ocasionada pelo depósito de materiais oriundos da construção da ETE em 2002.

O local apresenta topografia predominantemente plana (0 a 3\% de declividade), não estando suscetível à ação da erosão. Após a degradação, a área não havia recebido nenhuma medida de recuperação de solo, tampouco de revegetação, e apresentava cobertura vegetal escassa, com baixa possibilidade de regeneração natural (Figura 1A).

O delineamento experimental foi disposto em blocos casualizados com parcelas subdivididas, com dois tratamentos principais: presença de lodo de esgoto e ausência de lodo de esgoto, contendo três subparcelas com doses de lodo de ETA. Acrescentou-se um tratamento adicional, sem aplicação de lodos como testemunha.

As três doses de lodo de ETA corresponderam a 24, 37 e $61 \mathrm{Mg} \cdot \mathrm{ha}^{-1}$ de sólidos totais (ST), e a dose de lodo de ETE da parcela principal corres-

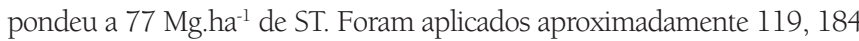

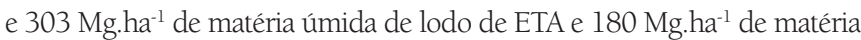
úmida de lodo de ETE, considerando o teor de umidade do material no dia da aplicação (de 79,9 e 57,4\%, respectivamente para o lodo de ETA e de ETE)

O experimento envolveu 7 tratamentos e 3 repetições, num total de 21 unidades experimentais com $40 \mathrm{~m}^{2}$ cada. As unidades experimentais foram dimensionadas com $5 \mathrm{~m}$ de largura por $8 \mathrm{~m}$ de comprimento, com $1 \mathrm{~m}$ de bordadura, além de uma faixa de $1 \mathrm{~m}$ como área de circulação e redução dos riscos de contaminação entre tratamentos.

O lodo utilizado no estudo foi gerado na ETA Passaúna, que possui vazão média de $1.685 \mathrm{~L} \mathrm{~s}^{-1}$ e cujo manancial de abastecimento é o Reservatório do Passaúna. A Tabela 1 apresenta resultados dos principais parâmetros de qualidade da água bruta afluente à ETA em 2007, ano em que foi gerado o lodo utilizado no estudo.

$\mathrm{Na}$ ETA Passaúna, a coagulação é realizada com $\mathrm{Al}_{2}\left(\mathrm{SO}_{4}\right)_{3}$ líquido ou Policloreto de Alumínio (PAC) e o lodo é gerado em 16 decantadores de alta taxa com lona. A remoção é hidráulica, sendo realizada alternadamente em dois decantadores por dia, mediante abertura de válvula no fundo do decantador. O lodo é adensado e desaguado em centrífuga com adição de polímero aniônico. A geração média é de $10 \mathrm{~m}^{3}$ de lodo a cada 10 horas.

O lodo utilizado no estudo, com granulometria de $93 \mathrm{~g} . \mathrm{kg}^{-1}$ de argila, 44 g. $\mathrm{kg}^{-1}$ de silte e 863 g. kg-1 de areia (textura "franco-arenosa", conforme Olenik et al., 2004), é proveniente exclusivamente dos decantadores, uma vez que a água de lavagem dos filtros retorna para o início do processo de tratamento da água bruta. 

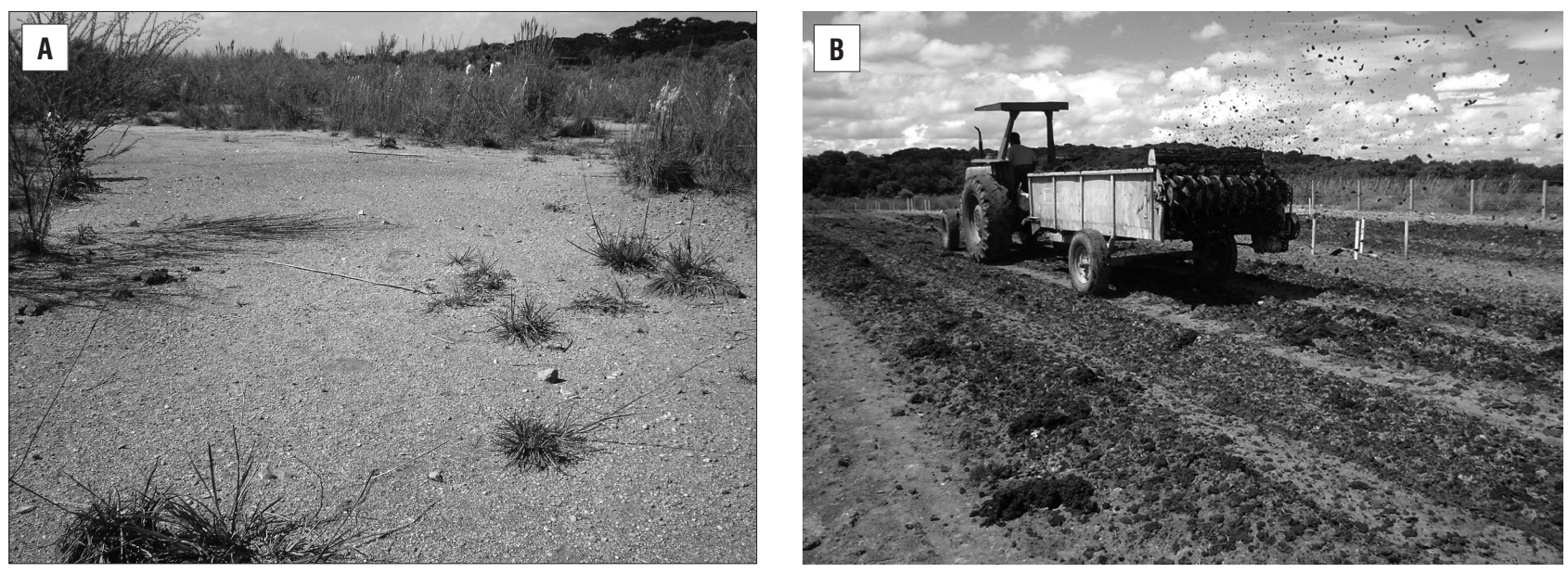

Figura 1 - Área degradada na Estação de Tratamento de Esgoto Padilha Sul, em Curitiba (PR), antes (A) e durante (B) a aplicação de lodos.

Tabela 1 - Resultados dos principais parâmetros de qualidade da água bruta afluente a Estação de Tratamento da Água Passaúna no ano de 2007.

\begin{tabular}{lcccc}
\hline Parâmetro & Unidade & Média & Máximo & Mínimo \\
\hline Turbidez & NTU & 2,5 & 6,3 & 0,3 \\
\hline Cor aparente & uH & 11,9 & 25,0 & 2,5 \\
\hline Alcalinidade total & $\mathrm{mg} \mathrm{L}^{-1} \mathrm{CaCO}_{3}$ & 53,4 & 59,0 & 40,0 \\
pH & - & 7,6 & 8,8 & 6,5
\end{tabular}

NTU: Unidades Nefelométricas de Turbidez; uH: unidade Hazen.

Já o lodo de esgoto foi produzido na própria ETE Padilha Sul, onde o tratamento é realizado por processo biológico anaeróbio, em reator anaeróbio de leito fluidizado (RALF). Ele foi desaguado em centrífuga, com adição de polímero catiônico e, posteriormente, higienizado por processo de estabilização alcalina prolongada, que consiste na adição de cal virgem a 50\% dos ST e armazenamento por 30 dias. O lodo foi mantido em armazém coberto, com laterais abertas, até o momento da aplicação, ou seja, aproximadamente 80 dias após a adição da cal.

Eles foram avaliados quanto aos parâmetros agronômicos, de sanidade e metais pesados, conforme estabelecido pelas Resoluções Conama 375/06 (BRASIL, 2006) e Sema 021/09 (PARANÁ, 2009). Os resultados da caracterização dos lodos utilizados no presente estudo, quanto ao potencial agronômico, são apresentados na Tabela 2.

A Tabela 3 mostra a caracterização dos lodos em relação a substâncias inorgânicas, comparativamente aos limites estabelecidos pela Resolução Sema 021/09 (PARANÁ, 2009) e pela Code of Federal Regulations (CFR) part.503, United States Environmental Protection Agency (EPA) para lodo de esgoto classe B com limites propostos para solos de áreas degradadas (ENVIRONMENTAL PROTECTION AGENCY , 1997).

As quantidades dos parâmetros inorgânicos adicionadas ao solo na aplicação das diferentes taxas de lodo de ETA e da aplicação de lodo de ETE encontram-se na Tabela 4.

Em relação à caracterização sanitária, os resultados dos atributos de ovos viáveis de helmintos e coliformes termotolerantes apresentaram-se compatíveis com a legislação vigente para ambos os lodos. No entanto, devido ao valor do atributo Salmonella sp, de $<3$ NMP $4 \mathrm{~g}^{-1}$ de ST para lodo de ETA e de $<2$ NMP $4 \mathrm{~g}^{-1}$ de ST para lodo de ETE estarem em níveis divergentes da legislação vigente, que estabelece ausência em $10 \mathrm{~g}$ de ST, foi realizado o monitoramento da presença do agente patogênico no solo após a aplicação dos tratamentos.

A área experimental tinha solo heterogêneo e antropogênico devido à deposição de diversos solos oriundos da construção da ETE, solos esses provenientes de uma região onde predominam os organossolos, cambissolos húmicos alumínicos e latossolos brunos ácrico (BHERING, 2008). Realizou-se a coleta de amostras compostas do solo das unidades experimentais a uma profundidade de 0,0 a 0,2 m, com a utilização de trado holandês. As amostras foram encaminhadas para laboratório para análise inicial de parâmetros agronômicos, físicos, microbiológicos e de substâncias inorgânicas. As médias, limites de confiança e coeficiente de variação dos resultados das análises de atributos químicos e granulométricos iniciais dos solos das 21 parcelas podem ser observados nas Tabelas 5 a 7 .

As etapas de preparo e manejo da área são apresentadas na Tabela 8. A aplicação dos lodos foi realizada, nas dosagens descritas anteriormente, com equipamento próprio para distribuição de materiais pastosos na agricultura (Figura 1B), sendo a operação realizada em três dias consecutivos, de modo a minimizar o erro experimental. Posteriormente, houve a incorporação por meio de aração a $0,20 \mathrm{~cm}$ de profundidade.

Aos 20 dias após a incorporação dos lodos, foi realizada a semeadura de $50 \mathrm{~kg} \cdot \mathrm{ha}^{-1}$ da espécie forrageira milheto (Pennisetum americanum CL, Leeke [P. Glaucum (L.) R. Br]), manualmente a lanço, com incorporação superficial ao solo. Aproximadamente 90 dias após o plantio, houve a colheita do vegetal e determinação da produção em matéria seca. As amostras foram retiradas de área total da unidade experimental e secas em estufa a $60^{\circ} \mathrm{C}$, até atingir peso constante.

Foram coletadas amostras compostas de solo em cada unidade experimental, a uma profundidade de 0,0 a 0,2 m, as quais foram analisadas para macro e micronutrientes, substâncias inorgânicas e sanidade. 
Tabela 2 - Potencial agronômico dos lotes de lodo.

\begin{tabular}{|c|c|c|c|}
\hline Parâmetro & Unidade & $\begin{array}{c}\text { Lodo na Estação de Tratamento de } \\
\text { Água Passaúna }\end{array}$ & $\begin{array}{c}\text { Lodo na Estação de Tratamento de } \\
\text { Esgoto Padilha Sul }\end{array}$ \\
\hline $\mathrm{pH}$ & - & 6,80 & 11,70 \\
\hline $\mathrm{ST}^{*}$ & g. $100 . g^{-1}$ & 20,10 & 42,60 \\
\hline N Kjeldahl & g. $100 . g^{-1}$ de ST & 0,68 & 0,73 \\
\hline $\mathrm{N}-\mathrm{NH}_{3}$ & g. $100 . \mathrm{g}^{-1}$ de ST & $3,3.10^{-3}$ & $4,0.10^{-3}$ \\
\hline $\mathrm{N}$ nitrito & g. $100 . \mathrm{g}^{-1}$ de ST & $<3,0.10^{-4}$ & $<6,0.10^{-4}$ \\
\hline $\mathrm{N}$ nitrato & g. $100 . \mathrm{g}^{-1}$ de ST & $<1,3.10^{-3}$ & $<3,0.10^{-3}$ \\
\hline $\mathrm{K}$ & g. $100 . \mathrm{g}^{-1}$ de ST & 0,06 & 0,19 \\
\hline $\mathrm{Ca}$ & g. $100 . \mathrm{g}^{-1}$ de ST & $<2,0.10^{-5}$ & 19,70 \\
\hline $\mathrm{Al}$ & g. $100 . \mathrm{g}^{-1}$ de ST & 6,40 & 0,09 \\
\hline $\mathrm{Fe}$ & g. $100 . \mathrm{g}^{-1}$ de ST & 4,11 & 2,33 \\
\hline S & g. $100 . \mathrm{g}^{-1}$ de ST & 0,29 & 0,32 \\
\hline $\mathrm{Na}$ & g. $100 . \mathrm{g}^{-1}$ de ST & 0,01 & 0,07 \\
\hline C org & g. $100 . \mathrm{g}^{-1}$ de ST & 2,30 & 10,00 \\
\hline $\mathrm{SV}^{*}$ & g. $100 . \mathrm{g}^{-1}$ de ST & 2,50 & 2,50 \\
\hline
\end{tabular}

ST: sólidos totais; SV: sólidos voláteis.

Tabela 3 - Resultados de parâmetros inorgânicos dos lotes de lodos comparados com os níveis de restrição para o estado do Paraná (Paraná, 2009) e Code of Federal Regulations Part 503 (Environmental Protection Agency, 1997).

\begin{tabular}{|c|c|c|c|c|c|c|c|c|c|c|c|c|}
\hline \multirow{2}{*}{ Substâncias inorgânicas } & As & $\mathrm{Ba}$ & $\mathrm{Cd}$ & $\mathrm{Cr}$ & $\mathrm{Cu}$ & $\mathrm{Hg}$ & $\mathrm{Mn}$ & Mo & $\mathrm{Ni}$ & $\mathrm{Pb}$ & $\mathrm{Se}$ & $\mathrm{Zn}$ \\
\hline & \multicolumn{12}{|c|}{$\mathrm{mg} \cdot \mathrm{kg}^{-1}$} \\
\hline Lodo Estação de Tratamento de Água & $<1,0$ & 106,3 & 8,0 & $<2,0$ & 32,5 & $<0,1$ & 649,0 & 15,3 & 5,8 & 30,2 & $<1,0$ & 80,5 \\
\hline $\begin{array}{l}\text { Lodo Estação de Tratamento de } \\
\text { Esgoto }\end{array}$ & 7,9 & 174,4 & 5,2 & $<2,0$ & 99,0 & 0,4 & 189,0 & 12,6 & 20,0 & 44,5 & 1,1 & 916,2 \\
\hline Limite Sema & 41 & 1.300 & 20 & 1.000 & 1.000 & 16 & - & 50 & 300 & 300 & 100 & 2.500 \\
\hline CRF Part. 503 & - & - & 85 & 3.000 & 4.300 & 57 & - & - & 420 & 840 & - & 7.500 \\
\hline
\end{tabular}

CFR Part.503 - Code of Federal Regulations part. 503.

Tabela 4 - Substâncias inorgânicas incorporadas ao solo com a aplicação das doses de lodo de Estação de Tratamento de Água e Estação de Tratamento de Esgoto em relação à carga acumulada teórica permitida pela Resolução Sema 021/09 (Paraná, 2009).

\begin{tabular}{|c|c|c|c|c|c|c|c|c|c|c|c|c|c|c|c|}
\hline \multirow{3}{*}{ Lodo } & \multirow{3}{*}{$\begin{array}{l}\text { Dose } \\
\text { (Mg.ha-1 }^{-1} \\
\text { de ST) }\end{array}$} & \multicolumn{14}{|c|}{ Carga aplicada } \\
\hline & & As & $\mathrm{Ba}$ & $\mathrm{Cd}$ & $\mathrm{Cr}$ & $\mathrm{Cu}$ & $\mathrm{Hg}$ & $\mathrm{Mn}$ & Mo & $\mathrm{Ni}$ & $\mathrm{Pb}$ & Se & $\mathrm{Zn}$ & $\mathrm{Fe}$ & Al \\
\hline & & \multicolumn{14}{|c|}{ kg.ha- } \\
\hline \multirow{3}{*}{$\begin{array}{l}\text { Estação de } \\
\text { Tratamento de } \\
\text { Água }\end{array}$} & 24 & $<0,02$ & 2,50 & 0,20 & $<0,05$ & 0,78 & $<2.10^{-3}$ & 15,58 & 0,37 & 0,14 & 0,73 & $<0,02$ & 1,93 & 985,50 & 1539,50 \\
\hline & 37 & $<0,04$ & 3,90 & 0,30 & $<0,07$ & 1,20 & $<4.10^{-3}$ & 24,02 & 0,57 & 0,21 & 1,12 & $<0,04$ & 2,98 & 1519,40 & 2373,40 \\
\hline & 61 & $<0,06$ & 6,50 & 0,50 & $<0,12$ & 1,98 & $<0,01$ & 39,61 & 0,93 & 0,35 & 1,84 & $<0,06$ & 4,91 & 2504,90 & 3912,90 \\
\hline $\begin{array}{l}\text { Estação de } \\
\text { Tratamento de } \\
\text { Esgoto }\end{array}$ & 77 & 0,61 & 13,40 & 0,40 & $<0,15$ & 7,62 & 0,03 & 14,54 & 0,97 & 1,54 & 3,43 & 0,09 & 70,55 & 1793,20 & 67,620 \\
\hline $\begin{array}{l}\text { Estação de } \\
\text { Tratamento } \\
\text { de Água + } \\
\text { Estação de } \\
\text { Tratamento de } \\
\text { Esgoto }\end{array}$ & $61+77$ & 0,67 & 19,90 & 0,90 & 0,27 & 9,60 & 0,04 & 54,15 & 1,90 & 1,89 & 5,27 & 0,15 & 75,46 & 4298,10 & 3980,60 \\
\hline Limite Sema 02 & $1 / 09$ & 30,00 & 265,00 & 4,00 & 154,00 & 137,00 & 1,20 & - & 13,00 & 74,00 & 41,00 & 13,00 & 445,00 & - & \\
\hline
\end{tabular}

Na continuidade da recuperação da área em estudo, realizou-se a cobertura de inverno com cultivo de aveia preta (Avena sp), azevém (Lolium multiflorum) e ervilhaca (Vicea sativa), sendo as plantas incorporadas ao solo 90 dias após a semeadura.

Em dezembro de 2008, após o nivelamento da área, as parcelas sem lodo de esgoto receberam adubação mineral com 10 kg.ha-1 de nitrogênio (N), $50 \mathrm{~kg} \cdot \mathrm{ha}^{-1}$ de pentóxido de fósforo
$\left(\mathrm{P}_{2} \mathrm{O}_{5}\right.$ e $50 \mathrm{~kg} \cdot \mathrm{ha}^{-1}$ de óxido de potássio $\left(\mathrm{K}_{2} \mathrm{O}\right)$ no momento da nova semeadura da espécie forrageira milheto (Pennisetum americanum CL, Leeke [P. Glaucum (L.) R. Br]). Aos 45 dias da semeadura, realizou-se a adubação de cobertura de $50 \mathrm{~kg}$.ha-1 ${ }^{-1}$ de $\mathrm{N}$ em todas as parcelas. A colheita foi realizada aos 90 dias, por meio de amostragem, selecionando-se $1 \mathrm{~m}^{2}$ em local padrão para todas as unidades experimentais. As amostras foram secas em estufa a 
Tabela 5 - Médias, limites de confiança e coeficiente de variação de atributos químicos do solo das 21 unidades experimentais.

\begin{tabular}{|c|c|c|c|c|c|c|c|c|c|c|c|c|}
\hline \multirow{2}{*}{ Parâmetros estatísticos } & \multirow{2}{*}{$\mathrm{pH}$} & $\mathrm{Al}$ & $\mathrm{H}+\mathrm{Al}$ & $\mathrm{Ca}$ & $\mathrm{Mg}$ & $\mathrm{K}$ & SB & $\mathrm{T}$ & $P$ & $\mathrm{C}$ & V & $\mathrm{m}$ \\
\hline & & \multicolumn{7}{|c|}{$\mathrm{cmol}_{\mathrm{c}} \mathrm{dm}^{-3}$} & mg.dm ${ }^{3}$ & g. $\mathrm{dm}^{-3}$ & \multicolumn{2}{|c|}{$\%$} \\
\hline Média & 4,04 & 6,65 & 16,02 & 1,18 & 1,65 & 0,09 & 2,92 & 18,94 & 1,58 & 9,37 & 15,20 & 69,50 \\
\hline Limite de confiança inferior (95\%) & 4,00 & 5,58 & 14,93 & 0,90 & 1,31 & 0,07 & 2,33 & 17,65 & 1,13 & 7,70 & 12,47 & 64,11 \\
\hline Limite de confiança superior (95\%) & 4,07 & 7,73 & 17,11 & 1,46 & 1,99 & 0,11 & 3,52 & 20,24 & 2,04 & 11,04 & 17,86 & 74,83 \\
\hline Coeficiente de variação (\%) & 1,75 & 35,36 & 14,93 & 51,16 & 44,65 & 45,05 & 44,65 & 14,97 & 62,97 & 39,00 & 38,98 & 16,93 \\
\hline
\end{tabular}

Tabela 6 - Médias, limites de confiança e coeficiente de variação de atributos químicos e granulométricos do solo das 21 unidades experimentais.

\begin{tabular}{lcccccccccc}
\multirow{2}{*}{ Parâmetros estatísticos } & Nkjeldhal & N amoniacal & Nitrito & Nitrato & Na & S & Fe & Areia & Silte & Argila \\
\cline { 2 - 10 } & \multicolumn{7}{c}{$\mathbf{g ~ k g}^{-1}$} \\
Média & 4,74 & 1,22 & 0,11 & 0,60 & 730,00 & 0,20 & 768,30 & 349,00 & 181,60 & 469,00 \\
Limite de confiança inferior (95\%) & 3,12 & 1,07 & 0,09 & 0,48 & 639,10 & 0,10 & 654,50 & 319,60 & 161,30 & 425,30 \\
Limite de confiança superior (95\%) & 6,36 & 1,37 & 0,13 & 0,73 & 820,90 & 0,20 & 882,10 & 378,10 & 202,00 & 513,70 \\
Coeficiente de variação (\%) & 74,82 & 27,28 & 34,50 & 46,62 & 27,30 & 45,70 & 32,50 & 18,39 & 24,59 & 20,65 \\
\hline
\end{tabular}

Tabela 7 - Médias, limites de confiança e coeficiente de variação de atributos químicos da camada superficial dos solos das 21 unidades experimentais e média de teores de horizonte B de solos da Região Metropolitana de Curitiba (Paraná, 2005).

\begin{tabular}{|c|c|c|c|c|c|c|c|c|}
\hline \multirow{2}{*}{ Parâmetros estatísticos } & $\mathrm{Ba}$ & $\mathrm{Cr}$ & $\mathrm{Cu}$ & Mn & Mo & $\mathrm{Ni}$ & $\mathrm{Pb}$ & $\mathrm{Zn}$ \\
\hline & \multicolumn{8}{|c|}{$\mathrm{mg} \mathrm{kg}^{-1}$} \\
\hline Média & 72,95 & 57,60 & 21,39 & 568,06 & 0,88 & 15,39 & 26,71 & 58,72 \\
\hline Limite de confiança inferior (95\%) & 44,31 & 47,72 & 16,06 & 325,63 & 0,63 & 13,67 & 23,73 & 45,72 \\
\hline Limite de confiança superior (95\%) & 101,58 & 67,61 & 26,72 & 810,49 & 1,14 & 17,10 & 29,69 & 71,72 \\
\hline Coeficiente de Variação (\%) & 86,08 & 37,82 & 54,66 & 93,57 & 64,02 & 24,50 & 24,46 & 48,55 \\
\hline $\begin{array}{l}\text { Média da Região Metropolitana de } \\
\text { Curitiba (Paraná, 2005) }\end{array}$ & 343,96 & 63,47 & 35,10 & 500,49 & 1,40 & 20,00 & 27,98 & 61,14 \\
\hline
\end{tabular}

Tabela 8 - Etapas de preparo e manejo da área no período de desenvolvimento do estudo.

\begin{tabular}{|c|c|c|c|c|c|c|c|}
\hline \multirow{2}{*}{ Etapas de preparo e manejo da área } & \multicolumn{6}{|c|}{2008} & \multirow{2}{*}{$\begin{array}{c}2009 \\
\text { jan-fev }\end{array}$} \\
\hline & jan-fev & mar-abr & mai-jun & jul-ago & set-out & nov-dez & \\
\hline 1. Aração & $\mathrm{x}$ & & & & & & \\
\hline 2. Gradagem de nivelamento & $x$ & & & & & & \\
\hline 3. Aplicação dos lodos & $x$ & & & & & & \\
\hline 4. Aração para incorporação dos lodos & $x$ & & & & & & \\
\hline 5. Semeadura de primeiro cultivo de milheto & $x$ & & & & & & \\
\hline 6. Colheita de primeiro cultivo de milheto & & & $x$ & & & & \\
\hline 7. Amostragem de solo & & & $\mathrm{x}$ & & & & \\
\hline 8. Semeadura de aveia, azevém e ervilhaca & & & & $x$ & & & \\
\hline 9. Incorporação das plantas ao solo & & & & & $x$ & & \\
\hline 10. Nivelamento da área & & & & & & $x$ & \\
\hline 11. Adubação mineral das parcelas sem lodo & & & & & & $x$ & \\
\hline 12. Semeadura de segundo cultivo de milheto & & & & & & $x$ & \\
\hline 13. Adubação de cobertura & & & & & & & $x$ \\
\hline 14. Colheita de segundo cultivo de milheto & & & & & & & $x$ \\
\hline
\end{tabular}

$60{ }^{\circ} \mathrm{C}$ até atingir peso constante e pesadas para a determinação da matéria seca.

O efeito dos tratamentos na área experimental foi avaliado por análise da variância. Utilizou-se o teste de Dunnett a 95\% de confiabilidade para comparar a média do tratamento testemunha com a média de cada um dos demais tratamentos, e teste de Tukey a 95\% de confiabilidade para comparação das médias na parcela principal e nas subparcelas. As análises estatísticas foram realizadas com o auxílio do software Assistat versão 7.5 beta (SILVA, 2008).

\section{Resultados e discussão}

\section{Características dos lodos}

O lodo da ETA Passaúna (Tabela 2) apresentou níveis semelhantes aos observados em lodo de ETA por Andreoli et al. (2003) para pH, potássio (K), Al, sódio (Na) e sólidos voláteis (SV); por Machado et al. (2004) para pH, N Kjeldahl e carbono orgânico (C org); por AWWA (1990) para N Kjeldahl, Al e C org; por Hoppen (2004) para K, Al e Na; e por Padilha (2007) para enxofre (S). 
Segundo Reali (1999), as características do lodo de ETA podem variar com o tipo de coagulante e auxiliares utilizados no tratamento e com a qualidade da água bruta, que apresenta alterações sazonalmente. Saron e Leite (2001) relatam que pode haver variação destas características também em função da tecnologia usada no tratamento de água.

Apesar do lodo de ETA empregado no experimento apresentar significativa quantidade de $\mathrm{Al}$ total (6,4\%, Tabela 2), sua aplicação nas dosagens adotadas: 24, 37 e $61 \mathrm{Mg} \cdot \mathrm{ha}^{-1}$ resultou em quantidades relativamente pequenas de Al total adicionadas ao solo, respectivamente de 641, 989 e $1.630 \mathrm{mg} \mathrm{kg}^{-1}$ de solo. Quando comparadas ao teor total médio, de $123.204 \mathrm{mg} \mathrm{kg}^{-1}$, dos solos paranaenses (PARANÁ, 2005).

O lodo da ETE Padilha Sul (Tabela 2) apresentou níveis semelhantes ao observado por Bittencourt et al. (2009) e Pires, Mattiazzo e Berton (2004) em lodos alcalinos de Curitiba e Jundiaí, respectivamente, quanto aos parâmetros de $\mathrm{pH}$ e ST. No entanto, os valores foram distintos para os parâmetros de $\mathrm{Al}$, ferro ( $\mathrm{Fe}$ ) e carbono orgânico (Corg). Segundo a Environmental Protection Agency (1997), Andreoli e Pegorini (2000) e Melo e Marques (2000), esse tipo de lodo apresenta composição variável em função das características do esgoto afluente, dos processos utilizados no sistema de tratamento de esgoto e de tratamento e higienização do lodo.

Os níveis dos parâmetros inorgânicos nos lodos apresentaram-se baixos (Tabela 3) quando comparados às quantidades estabelecidas pela legislação mais restritiva (Resolução Sema 021/09), sendo que as quantidades incorporadas ao solo (Tabela 4), inclusive na dose mais alta de lodo de ETA somada à dose de lodo de esgoto, apresentaram-se abaixo dos limites de carga acumulada teórica estabelecidos para lodo de esgoto pela Resolução Sema 021/09 (PARANÁ, 2009).

\section{Caracterização inicial do solo}

Em relação à fertilidade do solo, apesar da variabilidade existente na área (Tabela 5), os atributos analisados apresentaram, em sua maioria, níveis predominantes numa única faixa de classificação segundo Olenik et al. (2004). O nível de Al e a capacidade de troca de cátios (CTC) foram classificados em todas as unidades experimentais como altos, e a saturação em $\mathrm{Al}$ (m\%), muito alta, com exceção de uma, classificada como média. Os níveis de $\mathrm{Ca}$, Ke $\mathrm{P}$ corresponderam à classe baixa, com exceção de duas unidades experimentais para Ca e P e de seis para $\mathrm{K}$, classificadas como média. Já os níveis de Mg em 19 unidades experimentais corresponderam à classe alta e 2 à média. O parâmetro pH apresentou-se homogêneo, com coeficiente de variação baixo (1,75\%), classificado como acidez muito alta. A saturação de bases (V\%) indicou níveis de classe muito baixa, a não ser uma parcela com nível baixo, e o C teve comportamento heterogêneo com níveis na classificação baixa $(<8,0$ g.dm-3) e média $(8,0$ a 14,1 g.dm-3), sendo que apenas 2 parcelas classificaram-se com alta $\left(>14,1 \mathrm{~g} \cdot \mathrm{dm}^{-3}\right)$.

$\mathrm{Na}$ Tabela 6 verifica-se que a maioria das unidades experimentais apresentou, na camada superficial, solo de textura argilosa e franco-argilosa (320 a $516 \mathrm{~g} \mathrm{~kg}^{-1}$ de argila). Foram exceções apenas três parcelas: duas de textura Franca e uma de muito-argilosa, refletindo, juntamente com outros atributos como o Nitrogênio com coeficiente de variação de 74\%, na problemática do estudo de recuperação de áreas de depósito. Ainda na Tabela 6, observa-se que o Na apresentou valor médio inicial de 730 $\mathrm{mg} \mathrm{kg}$, o qual corresponde ao indicado para os solos da região onde está localizada a área experimental e 1,6 vez a média do Paraná (437 $\mathrm{mg} \mathrm{kg}^{-1}$ ), segundo Paraná (2005).

Os parâmetros Arsênio (As), Cádmio (Cd), Mercúrio (Hg), Selênio (Se) e Fenóis (Tabela 7) apresentaram-se no solo de todas as unidades experimentais abaixo do limite de detecção laboratorial (para $\mathrm{Cd}$, $\mathrm{Hg}$ e Se $<0,01 \mathrm{mg} \mathrm{kg}^{-1}$ e para As e Fenóis $<0,1 \mathrm{mg} \mathrm{kg}^{-1}$ ). Observa-se que a média de todos os parâmetros, com exceção do Manganês (Mn), foi abaixo da média verificada por Paraná (2005) para horizonte B de solos da Região Metropolitana de Curitiba (RMC).

\section{Produção vegetal}

O primeiro cultivo de milheto (Pennisetum americanum) apresentou desenvolvimento não uniforme, com falhas e crescimento heterogêneo em todas as unidades experimentais (Figura 2). O fato provavelmente foi ocasionado por impedimentos físicos e químicos do solo, resultantes da diversidade de materiais depositados na área. Outros fatores que possivelmente contribuíram foram: a semeadura do milheto no limite da data recomendada e a ocorrência de período de seca logo após a semeadura.

Desta forma, a produção de massa verde foi extremamente variável, com plantas de diversos tamanhos em uma única unidade experimental, gerando uma produção de matéria seca sem diferença significativa entre os tratamentos (Tabela 9).

No segundo cultivo de milheto, as plantas apresentaram crescimento mais uniforme, com maior altura e produção quando comparadas ao primeiro cultivo (Tabela 9). No entanto, houve problemas no desenvolvimento vegetal em unidades experimentais do primeiro bloco devido a um alagamento. Como verificado para o primeiro cultivo de milheto, apesar da realização de adubação nas parcelas sem lodo de esgoto, não foi registrada diferença significativa entre a testemunha e cada um dos tratamentos, bem como não houve interação entre os tratamentos da parcela principal (presença e ausência lodo de esgoto) e os tratamentos das subparcelas (doses de lodo de ETA).

\section{Caracterização final do solo}

Para os atributos de fertilidade do solo, aos 120 dias após a aplicação dos lodos não se verificou diferença significativa entre a testemunha e cada um dos tratamentos com doses crescentes de lodo de ETA (Tabela 10), demonstrando que o material não alterou as características do solo quanto a estes atributos. Os resultados aqui alcançados estão de acordo com as conclusões de Motta et al. (2005) quando afirmam que não há evidências de que o lodo de ETA possa acidificar o solo e aumentar o Al tóxico. Destacam-se para o caso em questão fatores como o período de avaliação e a quantidade deste tipo de lodo aplicada. 


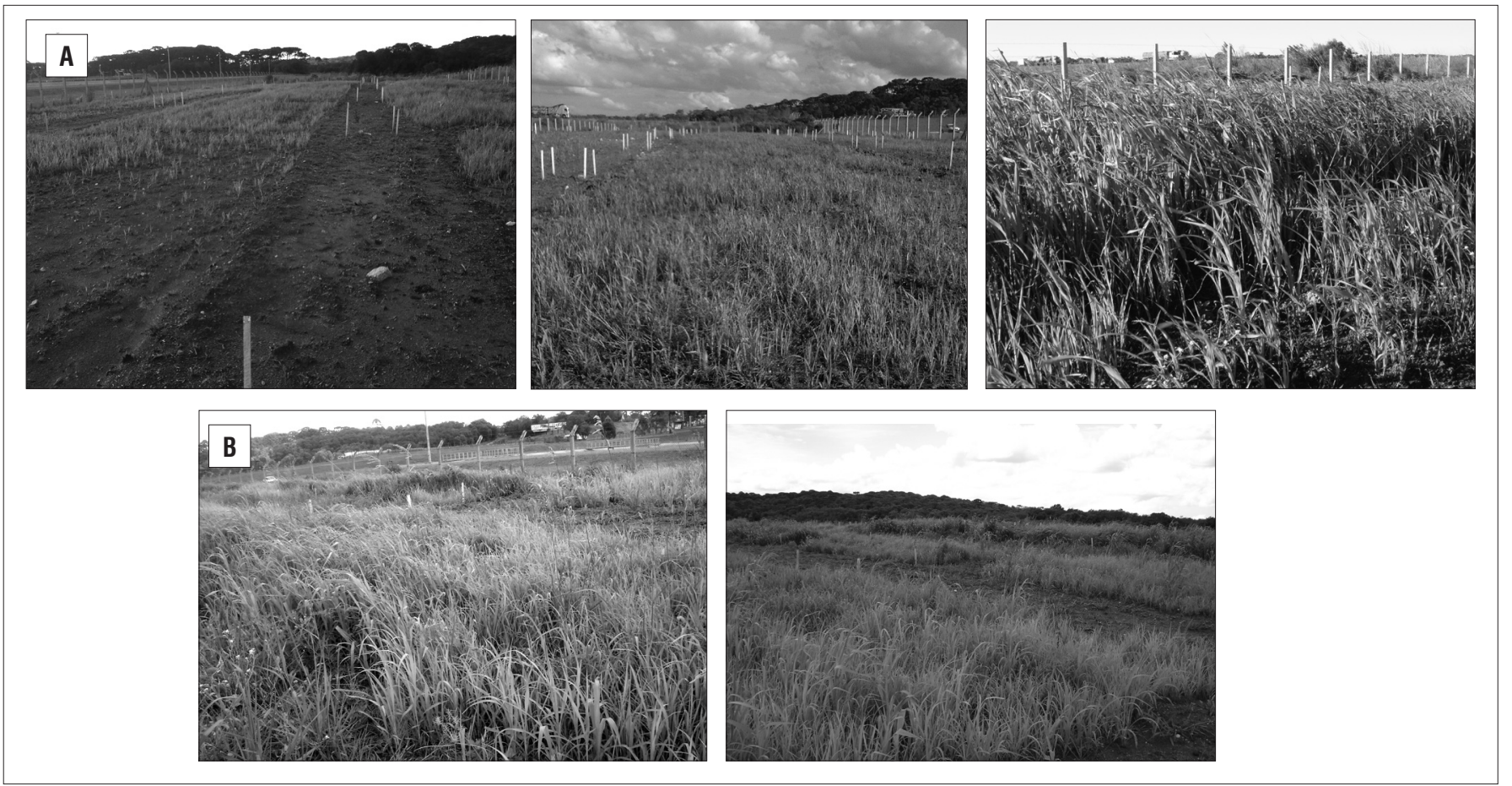

Figura 2 - Desenvolvimento do milheto aos 90 dias no primeiro (A) e no segundo cultivo (B).

Tabela 9 - Produção de milheto no primeiro e terceiro cultivo.

\begin{tabular}{lll}
\multirow{2}{*}{ Parâmetros estatísticos* } & \multicolumn{2}{c}{ Produção de milheto $\left(\mathrm{g} \cdot \mathrm{m}^{-2}\right.$ de MS) } \\
\cline { 2 - 3 } Média geral & \multicolumn{1}{c}{ Primeiro cultivo } & \multicolumn{1}{c}{ Segundo cultivo } \\
\hline Média máxima & 21,23 & 349,84 \\
\hline Média mínima & 12,27 & 479,87 \\
\hline $\begin{array}{l}\text { Diferença mínima signifi- } \\
\text { cativa }\end{array}$ & 28,72 & 248,27 \\
Coeficiente de variação (\%) & 55,6 & 505,13 \\
\hline
\end{tabular}

*No Teste de Dunnett, as médias não diferiram estatisticamente ao nível de 5\% de probabilidade.

Para o P (Tabela 11), embora AWWA (1995) comente que diversos autores verificaram redução da sua disponibilidade para alguns cultivos, em consequência da aplicação de lodo de ETA em solos agrícolas, esse fato não foi observado nas dosagens adotadas para o solo do presente experimento.

Os resultados dos atributos de fertilidade do solo nos tratamentos com doses de ETA acrescidos de uma única dose de lodo de ETE não tiveram sua comparação com a testemunha analisada, pois foram objeto de análise específica (parcela subdividida; Tabela 11), a qual permitiu testar possíveis interações. Desta forma, manteve-se o foco no objetivo desta pesquisa, ou seja, verificar o efeito do lodo de ETA na presença e ausência de lodo de ETE.

No modelo estatístico de parcelas subdivididas, para os atributos $\mathrm{pH}, \mathrm{P}, \mathrm{C}, \mathrm{Ca}, \mathrm{V} \%$ e $\mathrm{H}+\mathrm{Al}$ verificou-se diferença significativa entre os tratamentos da parcela principal (presença e ausência de lodo de esgoto) na comparação de médias por teste de Tukey (Tabela 11), não havendo diferença entre os tratamentos das subparcelas (doses de lodo de ETA), tampouco interação entre as parcelas principais e as subparcelas. Os resultados demonstram que, devido à composição do lodo de esgoto, rico em material alcalinizante e em matéria orgânica, sua aplicação na dosagem adotada alterou as características do solo quanto a esses atributos. Particularmente para o P, os resultados complementam o observado por Bittencourt et al. (2009) para lodo de esgoto da RMC constituído por quantidade significativa deste elemento (média de 0,63\% de $\mathrm{P}_{2} \mathrm{O}_{5}$ em 22 lotes).

Devido ao poder corretivo de acidez do lodo de esgoto utilizado, verificou-se que os teores de $\mathrm{Al}$ e $\mathrm{H}+\mathrm{Al}$ do solo decresceram nos tratamentos com aplicação do material. Porém, a diferença entre os tratamentos da parcela principal foi observada apenas para a variável acidez potencial (Tabela 11), enquanto para o Al não foi possível a comprovação estatística, em função da obtenção e repetição de valores iguais a zero nas unidades experimentais com lodo de esgoto, fato que impossibilitou a realização de análise para o modelo de parcelas subdivididas. Todavia, é importante observar que a quantidade de lodo de esgoto alcalinizado foi suficiente para neutralizar o Al tóxico, elevando em média o pH e a V\% acima de 5,4 e 80\%, respectivamente.

Para K, Mg e capacidade de troca de cátions ( $\mathrm{T}$ ) não houve diferença significativa entre os tratamentos principais no modelo parcelas subdivididas, indicando que o Ca em maior proporção contribuiu para a elevação do V\% e que o T manteve-se estável pela redução da $\mathrm{H}+\mathrm{Al}$.

Aos 120 dias após a aplicação dos tratamentos, os atributos químicos do solo - fenóis, Cd, Hg e Se — apresentaram-se, em todas as unidades experimentais, abaixo do limite de quantificação laboratorial (<0,1 mg kg-1 para fenóis; <0,01 mg kg-1 para Cd, Hg e Se), sendo que para o As somente 1 das 21 unidades experimentais apresentou-se acima do limite de quantificação $(<, 1)$, com valor igual a $0,7 \mathrm{mg} \mathrm{kg}^{-1}$, também abaixo do limite máximo permitido pela legislação. 
Tabela 10 - Comparação de médias entre a testemunha e cada um dos tratamentos com doses de lodo de Estação de Tratamento da Água na ausência e presença de lodo de esgoto*.

\begin{tabular}{|c|c|c|c|c|c|c|c|c|c|c|c|c|}
\hline \multirow[b]{3}{*}{ Tratamentos } & \multirow{2}{*}{\multicolumn{2}{|c|}{$\begin{array}{l}\text { Lodo (ST) } \\
\text { Mg.ha }^{-1}\end{array}$}} & \multicolumn{10}{|c|}{ Atributos de fertilidade do solo } \\
\hline & & & $\mathrm{pH}$ & $\mathrm{K}$ & $\mathrm{Ca}$ & $\mathrm{Mg}$ & $\mathrm{Al}$ & $\mathrm{H}+\mathrm{Al}$ & $\mathrm{T}$ & $\mathrm{C}$ & $\mathbf{P}$ & V \\
\hline & $\begin{array}{l}\text { Estação de } \\
\text { Tratamento de } \\
\text { Água }\end{array}$ & $\begin{array}{l}\text { Estação de } \\
\text { Tratamento de } \\
\text { Esgoto }\end{array}$ & $\mathrm{CaCL}_{2}$ & \multicolumn{6}{|c|}{$\mathrm{cmol}_{\mathrm{c}} \mathrm{dm}^{-3}$} & g.dm ${ }^{-3}$ & $\mathrm{mg} \cdot \mathrm{dm}^{-3}$ & $\%$ \\
\hline T1 Testemunha & 0 & 0 & $4,28 \mathrm{~b}$ & $0,15 a$ & $5,24 \mathrm{~b}$ & $2,28 \mathrm{~b}$ & 3,08 a & 9,51 a & $17,20 \mathrm{a}$ & $10,41 \mathrm{~b}$ & $6,71 \mathrm{~b}$ & $45,60 \mathrm{~b}$ \\
\hline T2 & 24 & 0 & $4,33 \mathrm{~b}$ & $0,15 a$ & $4,56 \mathrm{~b}$ & $2,20 \mathrm{~b}$ & $4,05 \mathrm{a}$ & $11,27 \mathrm{a}$ & $18,20 \mathrm{a}$ & $11,03 \mathrm{~b}$ & $5,43 \mathrm{~b}$ & $40,40 \mathrm{~b}$ \\
\hline T3 & 37 & 0 & $4,42 b$ & $0,11 \mathrm{a}$ & $4,31 \mathrm{~b}$ & $1,82 b$ & $2,66 \mathrm{a}$ & $10,71 \mathrm{a}$ & $16,90 \mathrm{a}$ & $11,75 b$ & $4,26 \mathrm{~b}$ & $36,60 \mathrm{~b}$ \\
\hline T4 & 61 & 0 & $4,28 \mathrm{~b}$ & $0,13 a$ & $3,65 \mathrm{~b}$ & $1,82 \mathrm{~b}$ & $3,30 \mathrm{a}$ & $11,40 a$ & $16,90 \mathrm{a}$ & $10,40 \mathrm{~b}$ & 5,46 b & $33,20 \mathrm{~b}$ \\
\hline T5 & 24 & 77 & $6,55 \mathrm{a}$ & $0,18 a$ & $15,97 \mathrm{a}$ & $4,17 \mathrm{a}$ & $0,00 \mathrm{a}$ & $2,33 \mathrm{~b}$ & $22,60 \mathrm{a}$ & $21,33 a$ & $12,17 \mathrm{a}$ & $89,60 \mathrm{a}$ \\
\hline T6 & 37 & 77 & $6,49 a$ & $0,19 a$ & $14,12 \mathrm{a}$ & $4,24 a$ & $0,00 \mathrm{a}$ & $2,36 \mathrm{~b}$ & $20,90 \mathrm{a}$ & $20,01 \mathrm{~b}$ & $18,93 \mathrm{a}$ & $88,40 a$ \\
\hline $\mathrm{T} 7$ & 61 & 77 & $5,74 a$ & $0,15 a$ & $12,15 \mathrm{a}$ & $3,57 \mathrm{a}$ & $0,12 \mathrm{a}$ & $3,73 \mathrm{~b}$ & $19,60 \mathrm{a}$ & $16,88 \mathrm{~b}$ & $14,10 a$ & $80,90 \mathrm{a}$ \\
\hline DMS & & & 0,99 & 0,06 & 5,46 & 2,36 & 4,38 & 7,87 & 9,66 & 7,69 & 5,76 & 34,45 \\
\hline CV (\%) & & & 7,93 & 17,50 & 26,21 & 33,80 & 95,44 & 44,12 & 27,30 & 33,01 & 12,50 & 23,89 \\
\hline
\end{tabular}

*De acordo com o Teste de Dunnett, letras iguais significam que a testemunha não diferiu estatisticamente dos outros tratamentos na mesma coluna, ao nível de $5 \%$ probabilidade. ST: sólidos totais; V: saturação de bases; DMS: diferença mínima significativa; CV: coeficiente de variação.

Tabela 11 - Comparação de médias entre os tratamentos da parcela principal para atributos de fertilidade do solo no modelo parcelas subdivididas.

\begin{tabular}{|c|c|c|c|c|c|c|c|c|c|c|}
\hline \multirow{3}{*}{ Tratamentos da Parcela Principal } & \multicolumn{10}{|c|}{ Atributos de fertilidade do solo } \\
\hline & \multirow{2}{*}{$\begin{array}{c}\mathrm{pH} \\
\mathrm{CaCL}_{2}\end{array}$} & $\mathrm{~K}$ & $\mathrm{Ca}$ & $\mathrm{Mg}$ & $A I^{* *}$ & $\mathrm{H}+\mathrm{Al}$ & $\mathrm{T}$ & \multirow{2}{*}{$\frac{C}{\mathrm{~g} \cdot \mathrm{dm}^{-3}}$} & \multirow{2}{*}{$\frac{\mathrm{P}}{\mathrm{mg} \cdot \mathrm{dm}^{-3}}$} & \multirow{2}{*}{$\begin{array}{l}\text { V } \\
\%\end{array}$} \\
\hline & & \multicolumn{6}{|c|}{$\mathrm{cmol}_{\mathrm{c}} \mathrm{dm}^{-3}$} & & & \\
\hline 0 Mg.ha-1 de lodo Estação de Tratamento de Esgoto & $4,35 b$ & $0,13 a$ & $4,17 \mathrm{~b}$ & $1,94 \mathrm{a}$ & 3,34 & $11,11 \mathrm{a}$ & $17,37 \mathrm{a}$ & $11,06 \mathrm{~b}$ & $5,05 \mathrm{~b}$ & $36,73 \mathrm{~b}$ \\
\hline 77 Mg.ha-1- de lodo Estação de Tratamento de Esgoto & $6,15 \mathrm{a}$ & $0,17 \mathrm{a}$ & $14,08 \mathrm{a}$ & 3,99 a & 0,04 & $2,81 \mathrm{~b}$ & 21,06 a & $19,41 \mathrm{a}$ & 15,06 a & $86,32 \mathrm{a}$ \\
\hline DMS & 1,29 & 0,07 & 4,14 & 2,47 & - & 5,70 & 3,82 & 1,17 & 6,20 & 23,28 \\
\hline CV (\%) & 12,14 & 23,16 & 22,37 & 40,94 & - & 40,31 & 9,79 & 3,80 & 30,36 & 18,64 \\
\hline
\end{tabular}

*Segundo o Teste de Tukey, letras iguais na coluna significam que as médias não diferiram estatisticamente ao nível de 5\% probabilidade.

** Análise estatística não realizada para o arranjo em parcelas subdivididas.

V: saturação de bases; DMS: diferença mínima significativa; CV: coeficiente de variação.

Para os demais elementos analisados (Tabela 12), não foi indicada diferença significativa entre a testemunha e cada um dos tratamentos com doses crescentes de lodo de ETA, demonstrando não haver efeito da aplicação do material nas características do solo quanto a esses atributos.

Observa-se, ainda na Tabela 12, que a média de todos os parâmetros, com exceção do Mn, apresentou-se abaixo da verificada por Paraná (2005) para horizonte B dos solos da RMC. Para alguns elementos, embora a testemunha apresente diferença dos tratamentos com lodo de ETA na presença de lodo de ETE, não permite a distinção dos efeitos individualizados e nem da interação. Assim, dos elementos citados na Tabela 12 , verificou-se efeito da interação entre os tratamentos aplicados apenas para os elementos N Kjeldahl, cobre (Cu) e molibidênio (Mo) (Tabela 13), ou seja, a resposta destes atributos do solo ao lodo de ETA aplicado variou com a presença ou ausência do lodo de ETE.

Dos resultados da Tabela 13, cabe ressaltar que os valores obtidos para o $\mathrm{N}$ apresentam um ponto de máxima, sugerindo aumento da atividade biológica na segunda dose de lodo de ETA quando na presença de lodo de ETE. Todavia, na maior dose, o valor médio do $\mathrm{N}$ foi novamente reduzido.

Isso sugere que, na presença do lodo de ETE, a aplicação de lodo de ETA foi favorável à dinâmica do $\mathrm{N}$ até a dose de $37 \mathrm{Mg}$.ha-1 ${ }^{-1}$, não interferindo na dose mais elevada.

\section{Conclusão}

O estudo da aplicação do lodo da ETA Passaúna em solo degradado, situado na RMC, na presença e ausência de lodo da ETE Padilha Sul, demonstrou que:

- não houve influência dos tratamentos no desenvolvimento vegetal do milheto;

- a aplicação do lodo de ETA não alterou as características do solo quanto às variáveis analisadas;

- na presença do lodo de esgoto, a aplicação de lodo de água foi favorá-

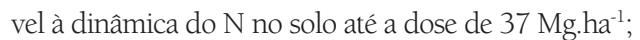

- a aplicação do lodo de ETE neutralizou o $\mathrm{Al}$ e alterou pH, $\mathrm{Ca}, \mathrm{H}+\mathrm{Al}$, C, $\mathrm{P} \mathrm{e} \mathrm{V \%}$ do solo.

Os resultados restringem-se às condições nas quais o estudo foi desenvolvido, considerando que características de água bruta distintas (concentração de algas, matéria orgânica, turbidez) afluente à ETA podem interferir em qualidade de lodo de ETA diferente da qualidade aqui apresentada.

Para designação da aplicação de lodo de ETA em solos como forma de destinação final do material, sugere-se a utilização do termo "aplicação controlada em solo", uma vez que, a aplicação na área objeto do presente estudo não apresentou potencial de melhoria nos atributos de fertilidade do solo. 
Tabela 12 - Comparação de médias de parâmetros inorgânicos dos solos entre a testemunha e os demais tratamentos e comparação com a média verificada por Paraná (2005).

\begin{tabular}{|c|c|c|c|c|c|c|c|c|c|c|c|c|c|c|}
\hline \multirow{3}{*}{$\begin{array}{l}\text { Trata- } \\
\text { mentos }\end{array}$} & \multicolumn{2}{|c|}{ Lodo } & \multicolumn{12}{|c|}{ Elementos químicos } \\
\hline & $\begin{array}{c}\text { Estação de } \\
\text { Tratamento } \\
\text { de Água }\end{array}$ & $\begin{array}{l}\text { Estação de } \\
\text { Tratamento } \\
\text { de Esgoto }\end{array}$ & $\mathrm{Ba}$ & $\mathrm{Cr}$ & $\mathrm{Cu}$ & $\mathrm{Fe}$ & $\mathrm{Mn}$ & Mo & $\mathrm{Na}$ & Nkjl & $\mathrm{Ni}$ & $\mathrm{Pb}$ & S & $\mathrm{Zn}$ \\
\hline & \multicolumn{2}{|c|}{ Mg.ha ${ }^{-1}$ ST } & \multicolumn{12}{|c|}{$\mathrm{mg} \mathrm{kg}^{-1}$} \\
\hline $\begin{array}{l}\text { T1Teste- } \\
\text { munha }\end{array}$ & 0 & 0 & $53,2 \mathrm{a}$ & $10,1 \mathrm{~b}$ & $25,0 \mathrm{~b}$ & 944,3 a & 513,3 a & $1,3 \mathrm{a}$ & 770,0 a & $2,6 b$ & $18,3 \mathrm{a}$ & $20,0 \mathrm{~b}$ & $0,1 \mathrm{a}$ & $49,2 \mathrm{a}$ \\
\hline $\mathrm{T} 2$ & 24 & 0 & $88,2 \mathrm{a}$ & $18,4 \mathrm{~b}$ & $18,2 \mathrm{~b}$ & 516,7 a & $457,1 \mathrm{a}$ & $3,8 \mathrm{a}$ & 735,0 a & $2,6 \mathrm{~b}$ & $23,3 a$ & $20,0 \mathrm{~b}$ & $0,1 \mathrm{a}$ & $36,0 \mathrm{a}$ \\
\hline T3 & 37 & 0 & $26,8 \mathrm{a}$ & $20,0 \mathrm{~b}$ & $26,5 b$ & 823,3 a & $381,5 \mathrm{a}$ & $0,5 a$ & $893,3 \mathrm{a}$ & $2,6 b$ & $23,3 a$ & $20,0 \mathrm{~b}$ & $0,2 \mathrm{a}$ & $84,0 \mathrm{a}$ \\
\hline T4 & 61 & 0 & $57,3 \mathrm{a}$ & $23,3 \mathrm{~b}$ & $22,5 b$ & $\begin{array}{c}1.091,7 \\
a\end{array}$ & 478,7 a & $0,9 a$ & 791,6 a & $3,8 \mathrm{~b}$ & $23,3 \mathrm{a}$ & $20,0 \mathrm{~b}$ & $0,2 \mathrm{a}$ & $67,5 \mathrm{a}$ \\
\hline T5 & 24 & 77 & $51,9 a$ & 50,0 a & $41,6 \mathrm{a}$ & $\begin{array}{c}1.116,7 \\
a\end{array}$ & $515,5 a$ & $0,6 a$ & 786,7 a & $6,0 \mathrm{a}$ & $43,3 a$ & $26,7 \mathrm{~b}$ & $0,1 \mathrm{a}$ & $268,8 \mathrm{a}$ \\
\hline T6 & 37 & 77 & $31,5 a$ & 27,7 b & $27,3 \mathrm{~b}$ & 899,7 a & 354,3 a & $1,0 a$ & $863,3 \mathrm{a}$ & $15,2 \mathrm{a}$ & $15,3 \mathrm{a}$ & $30,0 \mathrm{a}$ & $0,2 a$ & $88,9 a$ \\
\hline T7 & 61 & 77 & 69,4 a & $40,0 \mathrm{~b}$ & $36,9 \mathrm{~b}$ & $822,0 \mathrm{a}$ & $630,5 a$ & $1,8 a$ & 816,6 a & $2,9 \mathrm{~b}$ & $21,7 \mathrm{a}$ & $31,7 \mathrm{a}$ & $0,2 a$ & $111,4 a$ \\
\hline DMS & & & 91,6 & 31,8 & 13,7 & $1.438,7$ & 768,0 & 2,9 & 378,2 & 2,2 & 31,4 & 8,3 & 0,2 & 293,1 \\
\hline CV (\%) & & & 69,7 & 48,3 & 19,9 & 66,6 & 66,3 & 83,3 & 19,2 & 17,3 & 53,5 & 14,1 & 49,3 & 119,5 \\
\hline Média Mi & neropar RMC & Paraná, 2005) & 343,9 & 63,5 & 35,1 & $26.900,0$ & 500,5 & 1,4 & 639,8 & 700,0 & 20,0 & 28,0 & 11,0 & 61,1 \\
\hline
\end{tabular}

*Teste de Dunnett - letras iguais significam que a testemunha não diferiu estatisticamente dos outros tratamentos na mesma coluna, ao nível de $5 \%$ probabilidade. Zn: Zinco

Tabela 13 - Médias de interação entre os tratamentos da parcela principal e das subparcelas para elementos químicos do solo no modelo parcelas subdivididas.

\begin{tabular}{|c|c|c|c|c|c|c|c|c|c|}
\hline \multirow{3}{*}{$\begin{array}{l}\text { Tratamentos da parcela } \\
\text { principal [a] (ST) }\end{array}$} & \multicolumn{3}{|c|}{ N Kjeldahl } & \multicolumn{2}{|c|}{ Mo } & & \multicolumn{3}{|c|}{$\mathrm{Cu}$} \\
\hline & \multicolumn{9}{|c|}{ Tratamentos da subparcela [b] - Mg.ha ${ }^{-1}$ de lodo Estação de Tratamento de Água (ST) } \\
\hline & 24 & 37 & 61 & 24 & 37 & 61 & 24 & 37 & 61 \\
\hline $0 \mathrm{Mg}^{\mathrm{h}} \mathrm{ha}^{-1}$ de lodo ETE* & $2,60 \mathrm{bA}$ & $2,63 \mathrm{bA}$ & $3,80 \mathrm{aA}$ & $3,80 \mathrm{aA}$ & $0,50 \mathrm{aB}$ & $0,87 \mathrm{aB}$ & $18,17 \mathrm{bA}$ & 26,47 aA & $22,50 \mathrm{bA}$ \\
\hline $\begin{array}{l}77 \mathrm{Mg} \cdot \mathrm{ha}^{-1} \text { de lodo } \\
\text { ETE * }\end{array}$ & $6,00 \mathrm{aB}$ & 15,17 aA & $2,93 \mathrm{aC}$ & $0,60 \mathrm{bA}$ & $1,03 \mathrm{aA}$ & $1,83 \mathrm{aA}$ & $41,60 \mathrm{aA}$ & $27,30 \mathrm{aB}$ & $36,87 \mathrm{aAB}$ \\
\hline CV [a] \% & \multicolumn{3}{|c|}{20,10} & \multicolumn{3}{|c|}{90,23} & \multicolumn{3}{|c|}{25,30} \\
\hline CV [b] \% & \multicolumn{3}{|c|}{16,00} & \multicolumn{3}{|c|}{78,81} & \multicolumn{3}{|c|}{20,19} \\
\hline
\end{tabular}

ST: sólidos totais; ETE: Estação de Tratamento de Água;, CV: coeficiente de variação.

\section{Agradecimentos}

À SANEPAR, à Unidade de Serviço de Esgoto (USEG) e à Unidade de Serviço de Produção (USPD) da Região Metropolitana de Curitiba; e à Unidade de Serviço Processo Água (USAG) pelo apoio no desenvolvimento da pesquisa. Aos profissionais da ETE Padilha Sul, pela cessão dos equipamentos e contribuições nas atividades de campo, em particular a Samoel Ferreira Borges e Inácio de Andrade.

\section{Referências}

AMERICAN WATER WORKS ASSOCIATION - AWWA. (1990) Land application of water treatment sludge: impact and management. USA. AWWARF, $100 \mathrm{p}$.

AMERICAN WATER WORKS ASSOCIATION - AWWA. (1995) An assessment of cropland application of water treatment residuals. USA. AWWARF, $71 \mathrm{p}$.

ASSOCIAÇÃO BRASILEIRA DE NORMAS TÉCNICAS - ABNT. (2004) NBR 10004: Resíduos sólidos - classificação. Rio de Janeiro, 71 p.

ANDREOLI, C. V.; CARNEIRO, C.; PEGORINI, E. S. (2003) Projeto interdisciplinar de pesquisa sobre eutrofização de águas de abastecimento público na Bacia do Altíssimo Iguaçu: sub-projeto estudo das relações entre produção e composição do lodo gerado na estação de tratamento de água com parâmetros ambientais do lago Irai. FINEP
CT HIDRO. Curitiba, 23 p. Relatório técnico.

ANDREOLI, C.V. \& PEGORINI, E.S. (2000) Gestão pública do uso agrícola do lodo de esgoto. In: BETTIOL, W. \& CAMARGO, O.A. (EDS.). Impacto ambiental do uso agrícola do lodo de esgoto. EMBRAPA, Jaguariúna, SP, p. 281-312.

ASSOCIAÇÃO BRASILEIRA DE NORMAS TÉCNICAS - ABNT. (2004) NBR 10004: Resíduos sólidos - classificação. Rio de Janeiro, 71 p.

BARBOSA, A.B.D. (1997) A experiência da CAESB em recuperação de água de lavagem de filtros e desidratação de lodo de ETA. In: $19^{\circ}$ Congresso Brasileiro de Engenharia Sanitária e Ambiental, Anais... Foz do Iguaçu (PR): ABES. CD-ROM.

BHERING, S.B.; SANTOS, H.G. (2008) Mapa de solos do Estado do 
Paraná. Embrapa Florestas: Embrapa Solos: Instituto Agronômico do Paraná. Rio de Janeiro, 74 p.

BITTENCOURT, S.; ANDREOLI, C.V.; MOCHIDA, G.A.; SOUZA, L.M.K.M. (2009) Uso agrícola de lodo de esgoto, estudo de caso da Região Metropolitana de Curitiba. Revista Aidis, v. 2, n. 1, p. 1-11.

BRASIL. Ministério do Meio Ambiente. Conselho Nacional do Meio Ambiente. (2006) Resolução n 375, de 29 de agosto de 2006. Define critérios e procedimentos, para o uso agrícola de lodos de esgoto gerados em estações de tratamento de esgoto sanitário e seus produtos derivados. Brasília. Diário Oficial da União , n. 167, p. 141-146.

ENVIRONMENTAL PROTECTION AGENCY. (1997) Land application of biossolids: process design manual. Cincinnatti.

GRANDIN, S.R.; ALEM SOBRINHO, P. \& GARCIA JR., A.D. (1993) Desidratação de lodos produzidos em estações de tratamento de Água. In: XVII Congresso Brasileiro de Engenharia Sanitária e Ambiental, Anais... Natal (RN): ABES, v. 2, p. 324-341.

HOPPEN, C. (2004) Reciclagem de lodo de ETA centrifugado na construção civil, método alternativo para preservação ambiental. 135p. Dissertação (Mestrado em Engenharia de Recursos Hídricos e Ambiental) - Universidade Federal do Paraná, Curitiba.

MACHADO, L.C.G.T.; PEREIRA, J.A.R; PONTE, M.X.; LOPES, L.N.A. (2004) Avaliação do aproveitamento agrícola do lodo produzido na ETA Bolonha - RMB. In: ICTR 2004 -Congresso Brasileiro de Ciência e Tecnologia em Resíduos e Desenvolvimento Sustentável, Anais... Florianópolis (SC): Núcleo de informações de saúde ambiental da USP, p. 917-926.

MARQUES, D.M.L.M.; SILVA, A.P. \& BIDONE, F.R.A. (2000) Avaliação da lixiviação de alumínio e da produção de ácidos graxos voláteis em reatores anaeróbios utilizados para estudar a disposição final de lodos de ETAs em aterros sanitários. In: XXVII Congresso Interamericano de Engenharia Sanitária e Ambiental. Porto Alegre: AIDIS, p. 3-8. CD-ROM.

MELO, W.J.; MARQUES, M.O. (2000) Potencial do lodo de esgoto como fonte de nutrientes para as plantas. In: BETIOL, W.; CAMARGO, O.A. (ORGS). Impacto ambiental do uso agrícola do lodo de esgoto. EMBRAPA Meio Ambiente. Jaguariúna, p. 109-141.

PARANÁ. MINEROPAR. Minerais do Paraná S.A. (2005) Levantamento Geoquímico Multielementar do Estado do Paraná. Geoquímica de solo Horizonte B: Relatório final de projeto - Volume 2. Curitiba. Mineropar, 408 p.

MOREIRA, R.C.A.; GUIMARÃES, E.M.; BOAVENTURA, G.R.; MOMESSO, A,M.; LIMA, G.L. (2009) Estudo geoquímico da disposição de lodo de estação de tratamento de água em área degradada. Química Nova, v. 32, n. 8 , p. 2085-2093.

MOREIRA, R.C.A.; BOAVENTURA, G.R.; NUNES, S.A; PINHEIRO, L.A; NASCIMENTO, C. T. C.; SILVA, D.R.; LIRA, C.P. (2011) Geochemical and geophysical study in a degraded area used for disposal of sludge from a water treatment plant. Applied and Environmental Soil Science, v. 2011.

MOTTA, A.C.V.; HOPPEN, C.; ANDREOLI, C.V.; TAMANINI, C.R.; FERNANDES, C.V.S.; PEGORINI, E.S.; SOCCOL, V.T. (2005) Parecer técnico. Disposição final de lodos de estação de tratamento de água. Curitiba. UFPR, $43 \mathrm{p}$

OLENIK, J.; BRAGAGNOLO, N.; BUBLITZ, U.; SILVA, J.C.C. (2004) Análise de solo; tabela para transformação de resultados analíticos e interpretação de resultados. 3 ed. Curitiba. EMATER, p. 35.

PADILHA, J.C. (2007) Aplicação de lodos de tratamentos de água e esgoto em latossolos cultivados com milho e soja. 345p. Dissertação (Mestrado em Ciência do Solo) - Universidade Federal do Paraná Curitiba.

PARANÁ. Secretaria de Estado de Meio Ambiente e Recursos Hídricos (2009) Resolução SEMA 021/09. Dispõe sobre licenciamento ambiental, estabelece condições e padrões ambientais e dá outras providências, para empreendimentos de saneamento. Curitiba. Diário Oficial do Estado do Paraná, n. 7.962, p. 13-16.

PEDROZA, J.P.; HAANDEL, A.C.; BELTRÃO, N.E.M.; DIONÍSIO, J.A.; DUARTE, M.E.M. (2006) Qualidade tecnológica da pluma do algodoeiro herbáceo cultivado com biossólidos. Revista Brasileira de Engenharia Agrícola e Ambiental, v. 10, n. 3, p. 586-592.

PIRES, A.M.M.; MATTIAZZO M.E.; BERTON, R.S. (2004) Ácidos orgânicos como extratores de metais pesados fitodisponíveis em solos tratados com lodo de esgoto. Pesquisa Agropecuária Brasileira, v. 39, n. 7, p. 671-676.

REALI, M.A.P. (1999) Principais características quantitativas e qualitativas do lodo de ETAs. In: REALI, M.A.P. (COORD.) Noções gerais de tratamento de disposição final de lodos de ETA. Rio de Janeiro. ABES/ PROSAB, p. 21-39

SARON, A.; LEITE, V.M.B. (2001) Quantificação de lodo em estação de tratamento de água. In: $21^{\circ}$ Congresso Brasileiro de Engenharia Sanitária e Ambiental, Anais... João Pessoa (PB): ABES. CD-ROM.

SERRAT, B.M.; SANTIAGO, T.R.; BITTENCOURT, S; MOTTA, A.C.V.; SILVA, L.A.T.P.; ANDREOLI C.V. (2011) Taxa de aplicação máxima anual de lodo de esgoto higienizado pelo processo de estabilização alcalina: estudo comparativo de curvas de $\mathrm{pH}$ de solos. Revista Brasileira de Ciências Ambientais, n.19, p. 30-37.

SILVA, F.A.S. (2008) Programa computacional ASSISTAT - Assistência Estatística. Versão 7.5 beta. Disponível em: <http://www.assistat.com/ indformp.html>. Acesso em: mar 2009.

SOUZA, F.R. (2010) Compósito de lodo de estação de tratamento de água e serragem de madeira para uso como agregado graúdo em concreto. 209p. Tese (Doutorado em Ciência e Engenharia de Materiais) - Universidade de São Paulo, São Carlos.

TAMANINI, C.R.; MOTTA, A.C.V.; ANDREOLI, C.V.; DOETZER, B.H. (2008) Land reclamation recovery with the sewage sludge use. Brazilian Archives of Biology and Technology, v. 51, n. 4, p. 643-655.

TEIXEIRA, S.T;; MELO, W.J.; SILVA, E.T. (2005) Aplicação de lodo da estação de tratamento de água em solo degradado. Pesquisa Agropecuária Brasileira, v. 40, n. 1, p. 91-94. 